\title{
Effectiveness of Surveillance System in Detection of Tuberculosis in Sistan Region: What Is the Priority?
}

\author{
Mahdi Afshari ${ }^{{ }^{*}}$ \\ ${ }^{1}$ Department of Community Medicine, Zabol University of Medical Sciences, Zabol, Iran \\ *Correspondence to: Mahdi Afshari, mahdiafshari99@gmail.com
}

Received June 2, 2017, Accepted June 21, 2017, Published online June 30, 2018

\section{Dear Editor}

Tuberculosis (TB) is an infectious disease which is considered as the leading cause of death and a health concern worldwide. ${ }^{1}$ In 2016, the global incidence of TB was estimated as 10.4 million new (incident) cases, from which 6.2 million were men and $10 \%$ of them were infected with HIV. Countries with high burden of TB including India, Indonesia, China, Philippines, Pakistan, Nigeria, and South Africa are accounted for $64 \%$ of the new TB cases. ${ }^{2}$ The long common borders between Iran (Sistan and Baluchestan province) and Pakistan demonstrates the importance of this disease for Iranian community.

From an estimated 11500 TB cases (6100 males and 5400 females) in Iran in 2016, 9454 cases were identified rendering an estimated case detection rate $(\mathrm{CDR})$ of $80 \%{ }^{3}$ Among the Iran's provinces, Sistan and Baluchestan province has the highest TB incidence. Different factors are responsible for this high burden including border crossing of the Pakistani residents, ${ }^{4}$ and strong storms for half of the year. ${ }^{5}$

One of the targets within the third sustainable development goals (ensure healthy lives and promote well being for all ages) is: "By 2030, end the epidemics of AIDS, $\mathrm{TB}$, malaria and neglected tropical diseases and combat hepatitis, water borne diseases and other communicable diseases". To assess the plans and actions for controlling the TB incidence, estimating and monitoring the trend of $\mathrm{TB}$ incidence and relative indicators in different regions of Iran is necessary.

The most important methodological challenge defined in the Global Health Observatory (GHO) data is "determining the rate of under-reporting of cases from data facilities and/or routine surveillance systems". ${ }^{6}$ According to the reports of Iran's Center for Disease Control and Prevention (CDC), the notification rate of smear positive pulmonary TB in Sistan region (locating in the north of Sistan and Baluchestan province and the Zabol Medical University-covered area) has been decreased from 76.4 per 100000 population in 2011 to 42.9 per 100000 population in 2016. Such changes can be due to the reduction in the incident cases and the infection transmission within this high-prevalence region in response to the effective interventions as well as decreasing the environmental, behavioral, socioeconomic and nutritional risk factors. However, the most important problem is the real incidence of TB in this area which is required for calculating the CDR. To calculate the CDR, the notification rate should be divided by the real incidence rate. It demonstrates how much the TB control system is succeeded in identifying the TB cases within the community. During the last decade, WHO and Iran's CDC estimated the CDR more than $70 \%$. However, results of a cross sectional survey in 2013 showed that CDR in Sistan region might be much lower than those reported by the WHO. Therefore, further investigations are required to detect the real incidence and the CDR in this endemic area. A critical question based on the results of these studies is: "is the reduction in TB incidence during the last years due to the elimination of $\mathrm{TB}$ or is it a signal of low sensitivity of $\mathrm{TB}$ control system in finding the $\mathrm{TB}$ cases in Sistan region?"

To find real TB cases within the population, case finding should be improved. Previously, passive case finding was the only method of diagnosis of TB patients. From 2005 onwards, active methods were considered in Sistan and Baluchestan province. Active investigation of the prisoners, HIV positive cases, those with history of close contact, ${ }^{4}$ home to home investigations during vaccination campaigns, and frequent active case finding within the rural regions are examples of active methods. These strategies lead to early diagnosis and treatment of missed cases as well as determination of the real incidence of TB. Different methods have been suggested for estimating the TB incidence such as bootstrap, ${ }^{4}$ Styblo rules, ${ }^{7}$ and capture 
re-capture, ${ }^{8}$ These methods have been applied in different studies. However, scarce studies have been performed in Iran, particularly in Sistan and Baluchestan province. Due to the importance of TB control in Sistan region, the editorial board of the IJBSM invites all researchers to use numerous and intact information in this area for conducting relevant studies. Some research priorities in the field of TB in this area are as follows:

- Estimating the exact incidence and CDR of TB

- Active case finding

- Determining the deficiencies and challenges against the early diagnosis and treatment of TB patients

- Identifying high-risk populations

\section{Ethical Approval}

Not applicable.

\section{Competing Interests}

None.

\section{References}

1. Afshari M, Baneshi MR, Haghdoost AA. Comparing the results of cut-off method, anti-mode method and mirror image method in estimating the prevalence of tuberculosis infection. HealthMed. 2013;7(12):3103-3110.

Please cite this article as follows: Afshari M. Effectiveness of surveillance system in detection of tuberculosis in Sistan region: What is the priority? Int J Basic Sci Med. 2018;3(2):51-52. doi:10.15171/ijbms.2018.09.
2. Global tuberculosis report 2017. World Health Organization website. http://www.who.int/tb/publications/ factsheet_global.pdf.

3. Tuberculosis country profile 2016. World Health Organization web site. https://extranet.who.int/sree/ Reports?

4. Meysamie A, Salehi M, Sargolzaei N. Trend of Smear Positive Pulmonary Tuberculosis in Sistan and Baluchestan Province (2005-2008). Tanaffos. 2010;9(1):48-53.

5. Khazaei HA, Rezaei N, Bagheri GR, et al. Epidemiology of tuberculosis in the Southeastern Iran. Eur J Epidemiol. 2005;20(10):879-883. doi:10.1007/s10654-005-2152-y

6. Global Health Observatory (GHO) data. World Health Statistics 2017: Monitoring health for the SDGs. six lines of action to promote health in the 2030 agenda for sustainable development. http://www.who.int/gho/publications/ world_health_statistics/2017/EN_WHS2017_Part1.pdf.

7. Haghdoost AA, Afshari M, Baneshi MR, Gouya MM, Nasehi M, Movahednia M. Estimating the annual risk of tuberculosis infection and disease in southeast of iran using the bayesian mixture method. Iran Red Crescent Med J. 2014;16(9):e15308. doi:10.5812/ircmj.15308

8. Bassili A, Grant AD, El-Mohgazy E, et al. Estimating tuberculosis case detection rate in resource-limited countries: a capture-recapture study in Egypt. Int J Tuberc Lung Dis. 2010;14(6):727-732. 